\title{
Thermomechanical modeling of IN718 alloy directed energy deposition process
}

\author{
Adrien Doux ${ }^{1}$ and Vincent Philippe $^{1}$ \\ 1Digital Product Simulation, 1 Impasse M arcel Chalard, 31100 Toulouse, France
}

\begin{abstract}
Directed Energy Deposition (DED) Additive Manufacturing (AM) processes have a great potential to be used as cost-effective and efficient repairing and re-manufacturing processes for aerospace components such as turbine blades and landing gears. The AM OS project intends to connect repair and re-manufacturing strategies with design through accurate DED process simulation and novel multi-disciplinary design optimisation (MDO) methods. The ultimate goal is to reduce aerospace component weaknesses at design stage and prolong their lifecycles. DED AM processes are multi-physical phenomena involving high laser power melting powder or wire on a substrate. An experimental heat source has been calibrated using a heat transfer analysis of IN 718 laser and powder AM on a sample part. Residual stresses and final distortion are also computed using thermal field and the evolving part distortion at each increment. M ultiple hypotheses have been considered model the molten pool creation on the Heat A ffected Zone (HAZ).
\end{abstract}

\section{Introduction}

DED AM process simulation is extremely challenging. Multiple physical phenomena occur simultaneously such as fluid flow of blown powders, physics of heat generation due to laser beam, heat transfer into the AM part, and solid deformation. AM part is deposited on substrate at very high level of temperatures. A molten pool called Heat Affected Zone (HAZ) is locally created. Consequently, the global structure stiffness is modified due to residual stress introduction.

Process-induced stresses and distortions can be anticipated by Finite Element Analysis predictions. This paper proposes a thermomechanical approach using ABAQUS solver capabilities. An uncoupled heat transfer analysis which has been investigated is in reality a weak coupling thanks to the Element Progressive Activation method which accounts for geometrical non-linearity.

First, a complete thermal calculation computes thermal field in the entire part for each time increment. Then, thermal field is mapped at each increment of a stress analysis to determine final distortions and residual stresses. 


\section{Finite Element Analysis Assumptions}

DED process can be modelled as a thermomechanical analysis. The proposed study describes a simplified model using an uncoupled heat transfer analysis, rather than a fully coupled model. It enables in a first step to use a weak coupling thanks to Element Progressive Activation Method (available since ABAQUS 2018 release) which accounts for geometrical non-linearity. The approach assumes that mechanical and thermal solutions do not affect each other strongly.

The aim is to model the deposit of a multi-pass additive manufactured part. First, during heat transfer analysis, elements are progressively activated following a defined path during only one step. Then, resulting thermal field is mapped on the stress analysis mesh to compute process-induced distortions for each increment. Elements, for which the activation is turned on, are activated by assigning a volume fraction of material at the beginning of each increment.

Thus, the addition of material takes into account the current deformation of material. The partial element activation feature of ABAQUS is used to accomplish material addition during the simulation, as illustrated by Fig. 1.

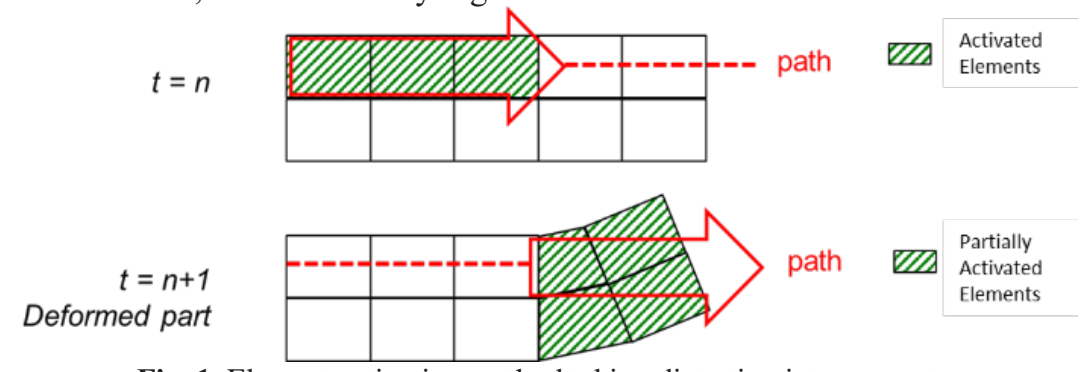

Fig. 1. Element activation method taking distortion into account

An automatic time increment scheme is used in all analyses. The maximum time increment was set to $100 \mathrm{~ms}$ for the stress analysis. The time step is reduced by automatic stepping scheme when necessary to prevent convergence difficulties.

\section{Model Definition}

Temperature data for thermal numerical model calibration were based on AMOS project inputs [1]. Wall geometric dimensions were $200 \times \times 19 \times 150 \mathrm{~mm}$ as illustrated in Fig. 2.

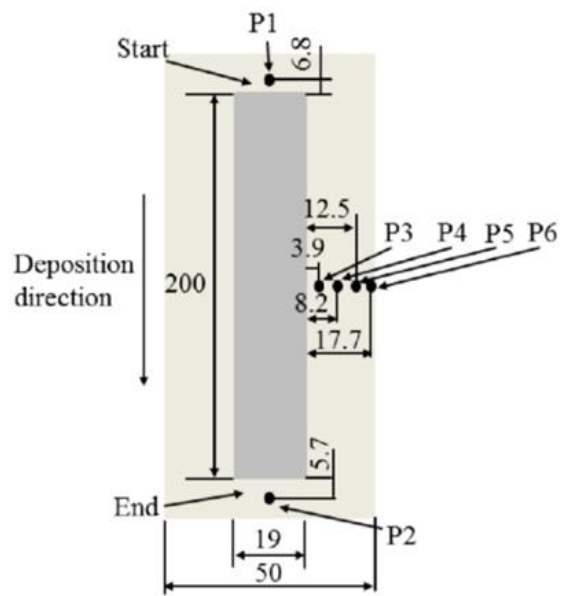

Fig. 2. Top view of the geometry dimensions of the wall and the thermocouple positions [1] 
A hexahedral continuum element mesh was considered regarding the deposited material and the substrate. A shell element mesh was used to assume a rigid surface behavior.

The hexahedral mesh size was set to $1 \mathrm{~mm}$ in order to minimize local effects during material deposition. The rigid surface mesh is coarser than the hexahedral according to master-slave surface ABAQUS requirements.

The analysis is assumed to be non-linear regarding large-displacements effects, material non-linearity and boundary non-linearity due to contact.

Laser heat source can be modelled as a Goldak double-ellipsoid [3] and improved the standard ball shape [2]. Deposit height, width and printing speed are imposed by the experiment. To obtain calibrated Goldak parameters, symmetry hypotheses were adopted $\left(c_{f}\right.$ $=c_{s}$ and $f_{f}=f_{r}$ ). $a$ and $b$ parameters were adjusted so that melt pool were as wide as the wall and as deep as the deposited material. Finally, power was adjusted to obtain the same maximum temperatures as the experiment.

Overall, heat source calibrated parameters were used for the analyses and are listed in Table 1.

Table 1: Parameters chosen for the building strategy

\begin{tabular}{|c|c|c|c|c|c|c|}
\hline $\begin{array}{l}\text { Heat source } \\
\text { type }\end{array}$ & $\begin{array}{l}\text { Bead Width } \\
\text { (mm) }\end{array}$ & $\begin{array}{l}\text { Bead Height } \\
\text { (mm) }\end{array}$ & $\begin{array}{c}\mathrm{a} \\
(\mathrm{mm})\end{array}$ & $\begin{array}{c}\mathbf{b} \\
(\mathrm{mm})\end{array}$ & $\begin{array}{c}\mathbf{c f} \\
(\mathrm{mm})\end{array}$ & $\begin{array}{c}\mathrm{cr} \\
(\mathrm{mm})\end{array}$ \\
\hline \multirow{3}{*}{ Laser } & 19.0 & 0.9 & 2.0 & 1.5 & 1 & 1 \\
\hline & $\begin{array}{l}\text { Heat source } \\
\text { efficiency }\end{array}$ & $\begin{array}{l}\text { Power } \\
(k W)\end{array}$ & \multicolumn{2}{|c|}{$\begin{array}{l}\text { Heat source speed } \\
(\mathrm{mm} / \mathrm{s})\end{array}$} & & \\
\hline & $90 \%$ & 6.0 & 5.7 & & & \\
\hline
\end{tabular}

The heat generated by the source induces a local effect below the laser application. The thermal effect due to energy is transmitted to the part by conduction, and is transferred to the environment by radiation and convection, except at the substrate bottom surface. A perfect emissivity of 1 , a film coefficient $\mathrm{h}$ of $0.5 \mathrm{~mJ} \cdot \mathrm{s}^{-1} \cdot \mathrm{mm}^{-2} .{ }^{\circ} \mathrm{C}^{-1}$ and a sink temperature of $20^{\circ} \mathrm{C}$ were used in the model. Substrate and deposited part are made of Inconel alloy 718 based on data from [4].

For the heat transfer analysis, the bottom surface was set to $20^{\circ} \mathrm{C}$. Regarding the stress analysis, the boundary conditions assumed a clamped substrate clamped on the rigid surface. Upon completion of the deposition process, the substrate is unclamped to release the internal stresses and reach a global equilibrium state. Both workpiece and substrate are subject to gravity.

Contact with the rigid surface is defined by a tangential behavior with 0.005 slip tolerance and a 0.5 friction coefficient. Normal behavior is controlled by a linear penalty method for enforcement of the contact constraint. Its stiffness is defined by a linear penalty stiffness of 0.1 .

\section{Experimental data for heat source calibration}

AMOS project contributed to experimental measurements from [1]. Six thermocouple positions (P1-P6) were placed on the substrate as detailed in Fig. 2.

Temperature data from [1] was used to calibrate the heat transfer analysis. The recorded temperature at the thermocouple locations are shown in Fig. 3. Only five out of six 
measurement points were chosen to compare experimental and numerical predictions: P1 and P2 at the substrate extremities; P3, P4 and P5 at the middle of the substrate. P6 was not considered due to schema imprecision.

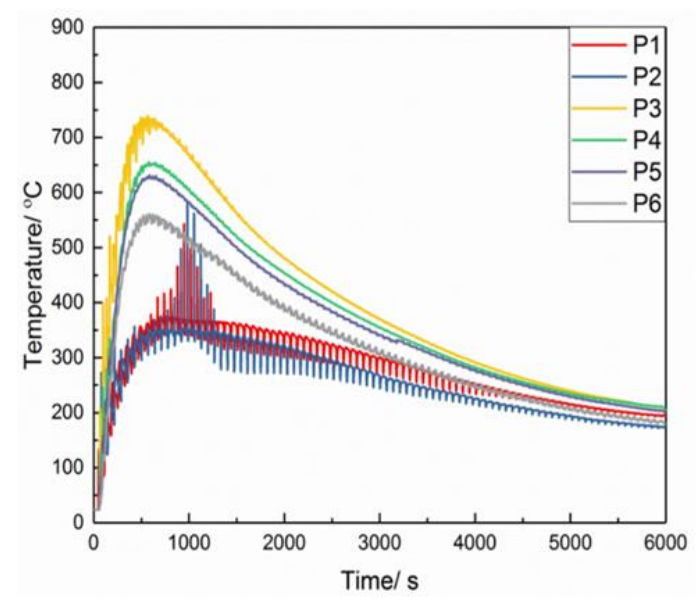

Fig. 3. Temperature profiles from experiment as a function of time [1]

High temperatures were recorded during the deposition of the first few layers, with important oscillations. This was attributed to the short time period between depositions, as the substrate did not have time to cool down completely before the deposition of subsequent layers.

Thus, the recorded temperatures increased until it reached a maximum at around $600 \mathrm{~s}$, corresponding to approximatively 15-20 passes. From that moment onwards, nozzle was high enough so that heat flux was smaller at the wall base. Consequently, the temperature profiles show decreasing temperatures until the end of the deposition process.

\section{Stress Analysis}

\subsection{Johnson-Cook's Implementation}

Johnson-Cook plasticity model is relevant for large strains, high strain rates and elevated temperatures, and is often used in adiabatic transient simulations. As a particular type of Mises plasticity, it expresses plastic yield stress as a function of plastic strain, strain rate, temperature, ambient temperature, reference strain rate, and material constants $A, B, C, m$ and $n$, as defined in Eq. $(1$.

$$
\sigma=\left(A+B \varepsilon^{n}\right)\left[1+C \ln \left(\frac{\dot{\varepsilon}}{\dot{\varepsilon_{0}}}\right)\right]\left[1-\left(\frac{T-T_{\text {room }}}{T_{\text {melt }}-T_{\text {room }}}\right)^{m}\right]
$$

Three material behaviors are uncoupled in three terms. The first one depicts strain hardening; the second one represents viscosity or strain rate dependency, and the last one models thermal softening. The latter is voided when the temperature equals the melting temperature, so there is no plastic stress, as the material has melted. Yield stress is given as a function of plastic strain. This model is convenient since it takes into account a reasonable number of parameters, which are identified thanks to mechanical experiments. 


\subsection{Alternative Johnson Cook's Expression}

Iturbe et al. [5] proposed a modified expression to compute plastic yield in order to consider new material effects (Eq.(2). A strain-dependent exponential is introduced into the first term to account for strain softening. An exponential temperature dependency of the $C$ constant is added in the viscosity term.

$$
\sigma=\sigma_{\varepsilon}(\varepsilon) \sigma_{T}(T) \cdot \sigma_{\dot{\varepsilon}}(\dot{\varepsilon}, T)
$$

The yield stress from the modified Johnson-Cook plasticity model is illustrated in Fig. 4. Strain softening is taken into account so that the equivalent von Mises stress decreases above 0.3 equivalent plastic strain.

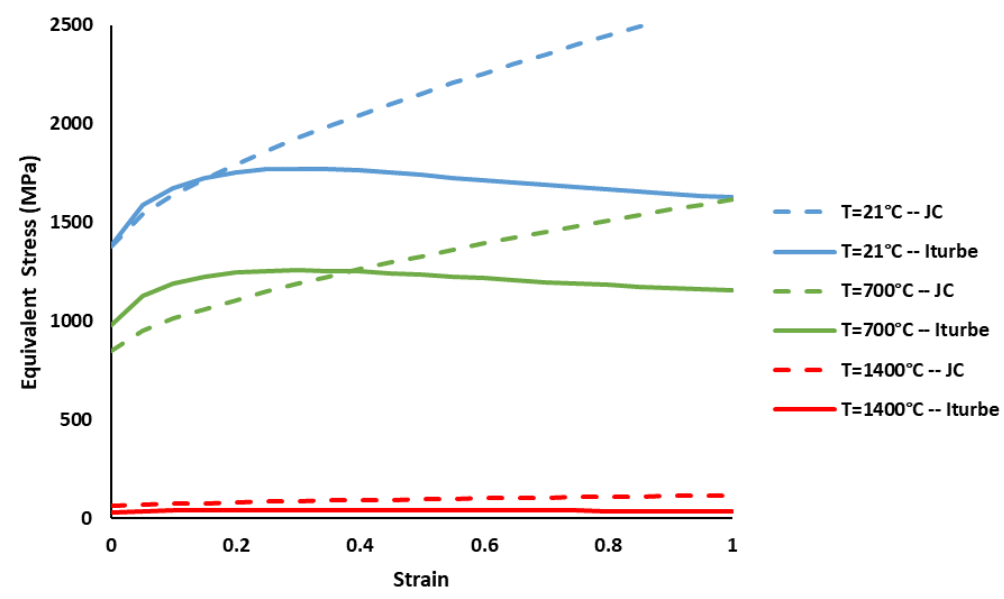

Fig. 4. Iturbe's modified Johnson-Cook yield stress (M Pa) at different temperature, $\dot{\varepsilon}=\dot{\varepsilon_{0}}$

\section{Results and Discussions}

Concerning heat source calibration, each layer deposition was simulated in a single pass. The melt pool was assumed to cover the entire wall width to be able to manufacture the part. As illustrated in Fig. 5, the melt pool in grey was large with a maximum depth of $2 \mathrm{~mm}$.

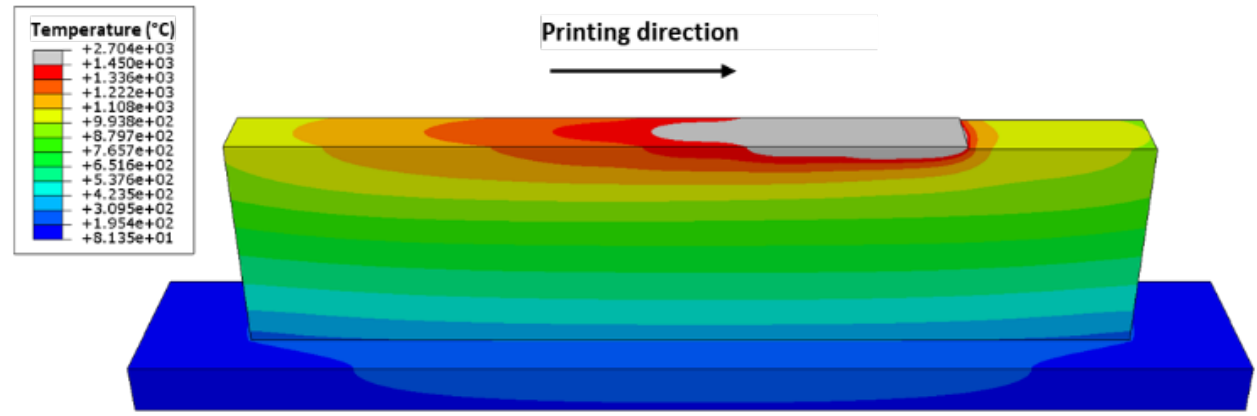

Fig. 5. Thermal field during DED process heat transfer analysis 
After the calibration of the heat source parameters, the predicted temperatures were plotted at two positions corresponding to thermocouples in the experiment. The obtained profile is presented in Fig. 6.

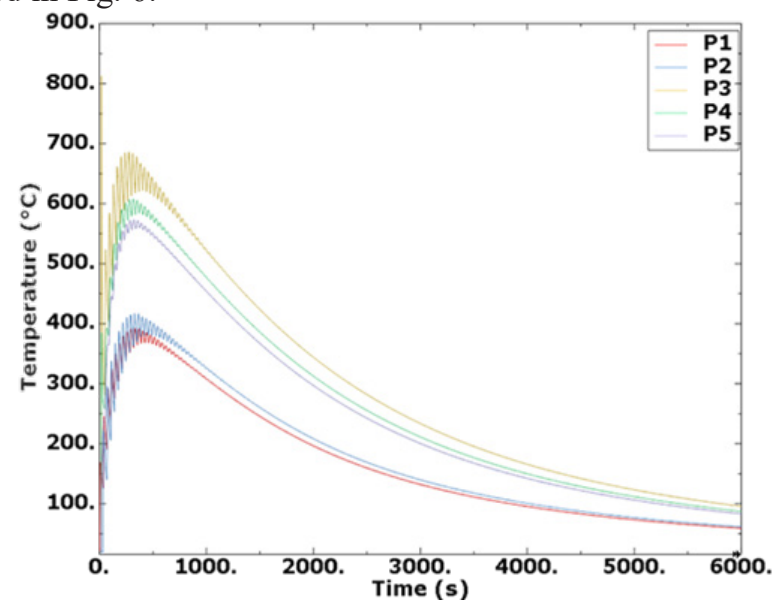

Fig. 6. Temperature profile from simulation as a function of time

The temperature profile from simulation gave an accurate prediction of the experimental results. The comparison is summarized in Table 2.

Table 2: Comparison of principal heat source calibration results

\begin{tabular}{|c|c|c|c|c|}
\hline & $\begin{array}{l}\text { P1 Peak } \\
\left({ }^{\circ} \mathrm{C}\right)\end{array}$ & $\begin{array}{c}\text { P4 Peak } \\
\left({ }^{\circ} \mathrm{C}\right)\end{array}$ & $\begin{array}{c}\text { Final Temperature } \\
\left({ }^{\circ} \mathrm{C}\right)\end{array}$ & $\begin{array}{l}\text { Oscillations } \\
\text { After 1000s }\end{array}$ \\
\hline Experiment & $400-450$ & 650 & 200 & Yes \\
\hline Simulation & 400 & 600 & 100 & No \\
\hline
\end{tabular}

The overall temperature profile was similar, with a significant temperature increase during the first dozen of passes, followed by a gradual decrease in temperature over a time period of $4000 \mathrm{~s}$.

The predicted temperature at point $\mathrm{P} 4$, located at the middle distance from the two extremities peaked at around $600^{\circ} \mathrm{C}$ instead of $650^{\circ} \mathrm{C}$ in the experiment. P1 situated at substrate extremity peaked at $400^{\circ} \mathrm{C}$. The large experimental oscillations for $\mathrm{P} 1$ around $1000 \mathrm{~s}$ were predicted by the numerical model. The predicted temperature after 6000 s was underestimated by $100^{\circ} \mathrm{C}$ compared to the experimental temperature recorded at the same time.

The thermal field from heat transfer analysis was mapped onto each increment of the stress analysis. Material non-linearity incorporated into the model includes temperature dependence of elastic modulus, coefficient of thermal expansion, thermal conductivity and specific heat capacity. The molten pool is created on the HAZ, where phase changes from solid to liquid occur when the temperature exceeds melt temperature. For finite element simulation purposes, material behavior has been assumed to follow a viscoplastic rule. For temperatures above the melting point, the Young's modulus is assumed to be zero.

Regarding the stress analysis, the predictions of stress and displacement from the modified Johnson-Cook plasticity model were compared with predictions from the classic Johnson-Cook model. An ABAQUS user subroutine called UHARD was implemented to compute yield stress as described by Eq. (1 at each increment. 
Predictions of Von Mises stresses and global displacements for both plasticity models were compared in Fig. 7 and Fig. 8 respectively. For computational efficiency, only seven additive manufacturing passes were simulated.

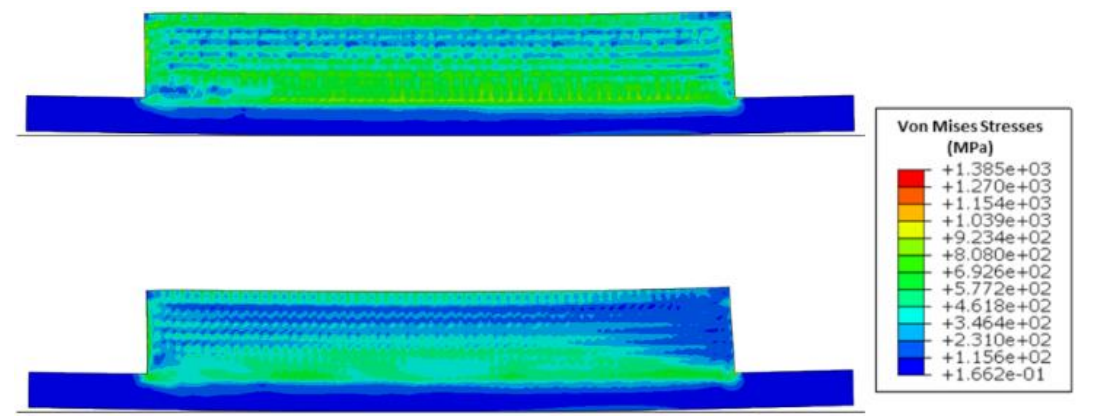

Fig. 7. V on M ises stresses (M Pa) comparison between tabulated J ohnson-C ook law (top) and Iturbe's modified behavior (bottom)

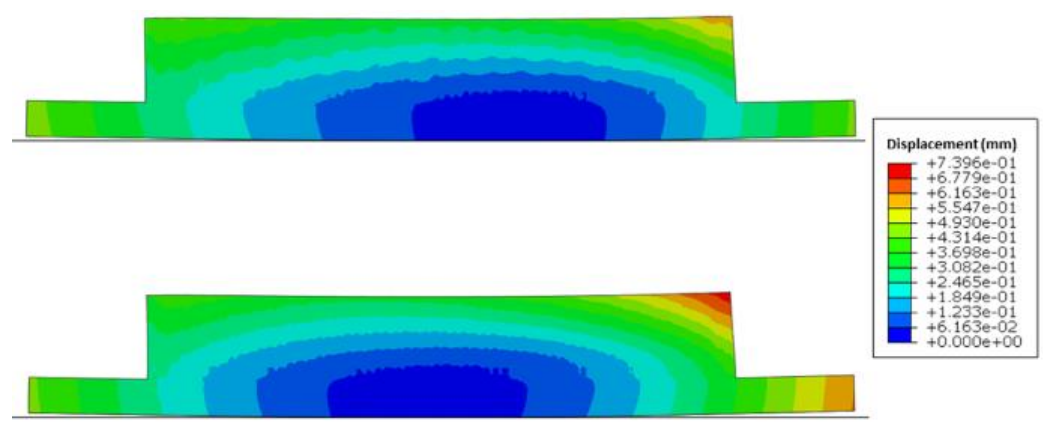

Fig. 8. Distortion (mm) comparison between tabulated J ohnson-Cook law (top) and Iturbe's modified behavior (bottom)

During the deposition section of the analysis, the substrate borders are maintained against the rigid surface using $100 \mathrm{MPa}$ uniform pressure to simulate clamping. After deposition, a cooling time of $600 \mathrm{~s}$ is applied before the flanges are released.

Global Von Mises stress level is significant for both material law with around $800 \mathrm{MPa}$. Yield strength is exceeded for some elements because of Inconel alloy 718 yield strength $(0.2 \%$ offset $)$ is equal to $1 \mathrm{GPa}$ at room temperature. Heat treatments enable to reduce residual stresses after printing and cooling.

In comparison, Von Mises stress prediction from the modified Johnson-Cook plasticity model is lower than the stresses computed with classic Johnson-Cook model, owing to strain rate hardening assumption.

With regards to distortion, after the clamps are released, a characteristic bended profile is predicted. However, it is more pronounced using the modified Johnson-Cook model. For example, on the right side, substrate displacement is $0.62 \mathrm{~mm}$ high with the modified plasticity model, i.e. $40 \%$ more than what Johnson-Cook predicts. Maximum displacement in the top right corner is also more noticeable with Iturbe's model. It corresponds to the last laser position, as it goes from left to right in the model. 


\section{Conclusions}

DED process simulation has been modelled using a thermomechanical approach. The heat source was successfully calibrated using experimental data from [1] despite thermal maxima under-estimation.

Regarding the stress analysis, very high level of stresses can result from uncoupled heat transfer analysis assumptions. Temperature mapping is assumed not to be influenced by stress analysis. However, a fully coupled analysis, which is not available so far using EPA on ABAQUS solver, may consider effects of phase transformations. Volumetric changes, density and cooling strain dependency may be considered so that thermal field is incrementally impacted. However, very high temperature data should be investigated deeper before validating stress predictions. Inconel alloy 718 material data for very high temperatures should be improved to predict accurate residual stresses and final distortion.

However, two different solutions were implemented - a conventional Johnson-Cook plasticity model and a modified one - but they are not comparable. Experimental studies of distortion from three recent coupons are anticipated in the near future and these results will be used for model validation studies.

\section{References}

1. W. Guo et U. Woy, Ti6Al4V and Inconel 718 DED thermal models (2017).

2. X. Jia et et. al., A New Method to estimate heat source parameters in gas metal arc welding simulation process, (Fusion Engineering and Design, pp. 40-48, Volume 89, 2014).

3. J. Goldak et et. al., A new finite-element model for welding heat-sources, (Metall. Trans., pp. 299$305,1985)$.

4. Special Metals Corporation, INCONEL Alloy 718 (2007).

5. A. Iturbe et et al., Mechanical characterization and modelling of Inconel 718 material behavior for machining process assessment (Materials Science and Engineering, pp. 441-453, 2017). 